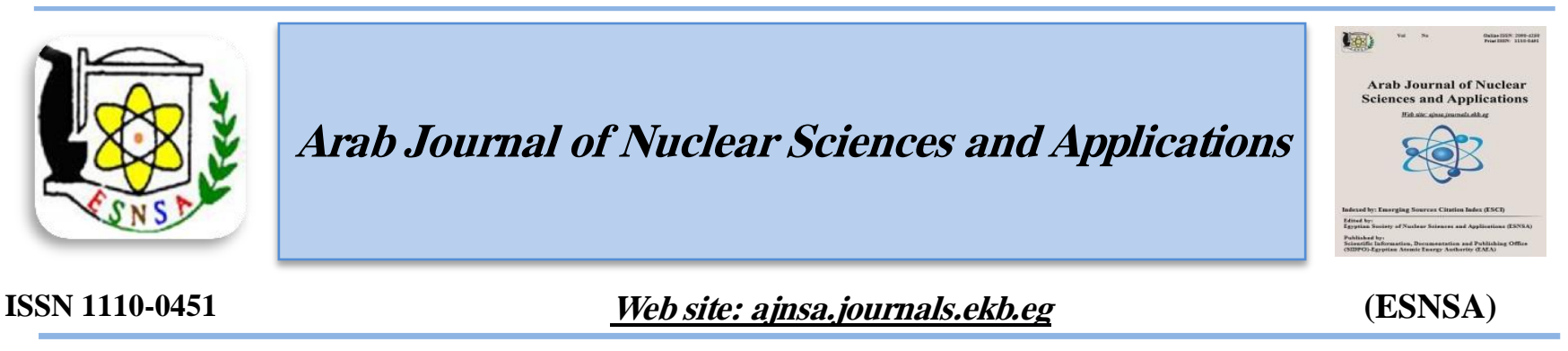

\title{
Towards a Database for the Relative Spectral Lines' Intensities of Laser- Induced Breakdown spectra
}

\author{
A. H. Galmed \\ National Institute of Laser Enhanced Science (NILES), Cairo University, Egypt
}

Received $3^{\text {rd }}$ Apr. 2019 The relative intensity is one of the parameters of the spectral lines that indicate the emission strength of Accepted $22^{\text {th }}$ Aug. $\quad$ such lines. The spectral lines relative intensity database for most elements has been recorded a long time ago through spectroscopic data obtained by arc, spark, and vacuum tube. The problem is that relative line intensity is source dependent and the current database cannot be used for Laser-Induced Breakdown Spectroscopy (LIBS). Since LIBS is dealing with transient plasma, then several parameters should be studied in order to find suitable conditions and a suitable way to record this database.

In this work, the author is trying to find a way to construct a database for relative lines intensities that can be used properly in LIBS analysis. The LIBS spectral lines have been recorded for different delay times for different copper alloy samples. Then, some chosen copper' spectral lines were compared. An empirical formula has been proposed to calculate the spectral line intensity for each delay time. It was found that for delay times between 1000 and $2000 \mathrm{~ns}$, the spectral lines were related together in a way that the copper concentration doesn't affect the spectral line relative intensity, but it only affects the value of the spectral line intensity.

Keywords: LIBS / Database / Relative intensity / Quantitative analysis

\section{Introduction}

The simplicity, rapidness and the no necessity for sample preparation in LIBS, as a well-known spectrochemical analytical technique [1,2], made it practical and attractive technique in many applications [3-6]. The data analysis for LIBS is simple and fast as long as it is used in the qualitative and semi-quantitative analysis but becomes harder and slower when the quantitative analysis is aimed. This is due to the precautions to be taken and the methods of analysis that are used [7].

For quantitative LIBS analysis, there are two main known methods. In the first method, which depends on data calibration, a set of standard samples having a matrix similar to that to be analyzed, are prepared and their spectral lines are used to construct calibration curves for each contained element. Thereafter, the unknown sample spectral lines intensity is measured and the concentration of each element is calculated from its pre-prepared calibration curve. In order to minimize some experimental fluctuations as well as the matrix effects, which could affect the calibration graph linearity, emission line intensity should be normalized to another line of an element of normal concentration. This element can be the most abundant element in the sample or carbon in the atmosphere. In some works, the LIBS spectrum is normalized to the spectral background signal [8]. Recently, some other methods for data calibration that are based on multivariate calibration

Corresponding author: ahmedgalmed@niles.edu.eg

DOI: 10.21608/ajnsa.2019.11482.1200

(C) Scientific Information, Documentation and Publishing Office (SIDPO)-EAEA 
techniques, like Partial Least Squares regression (PLS), Principal Components Analysis (PCA) and Artificial Neural Networks (ANN), are used for LIBS quantitative analysis [9-15]. These methods can give some important information that cannot be obtained from the ordinary calibration curve.

The second method is the Calibration Free (CF) method, proposed by Ciucci el al. [16]. This method is based on the LTE assumption and on calculating the exact excitation temperature from the Boltzmann plot line slope and the total number density of the given species $\left(\mathrm{N}_{0}\right)$ from the intercept. Then $\mathrm{N}_{0}$ is proportional to the species weight percentage in the sample $[7,16]$.

For the quantitative LIBS analysis to be carried out by the CF method, some assumptions should be fulfilled. These include stoichiometric ablation, LTE condition, plasma homogeneity, and optical thin plasma. CF LIBS is the best method for LIBS analysis in case of lack of calibration curves although the accuracy of CF is critically dependent on the conditions mentioned. [17].

It is believed that if a database is available for the relative intensity of the LIBS spectrum for each element and to study the effect of the surrounding parameters (experimental and plasma parameters), this will facilitate constructing standard calibration curves for each element, and hence it will lead to finding a new simple way for quantitative LIBS analysis.

The aim of the present work is to find the relative intensity of some $\mathrm{Cu}$ lines relative to each other that can be considered as an indicator for the LIBS users.

\section{Experimental Setup:}

The laser-induced plasma was produced by focusing the fundamental wavelength $(1064 \mathrm{~nm})$ of the Nd: YAG laser $(72 \mathrm{~mJ}, 6 \mathrm{~ns})$ onto the surface of certified copper samples (S160, S162 and S164) [Table 1] in air at atmospheric pressure via a quartz planoconvex lens of $10 \mathrm{~cm}$ focal length. The fluence was $35.4 \times 10^{6} \mathrm{~J} / \mathrm{m}^{2}$ in the focus of the laser light. The plasma emission was collected using a quartz optical fiber of a $600-\mu \mathrm{m}$ core diameter.

The optical fiber was aligned with the center of the plasma plume to keep the emission-signal collection perpendicular to the plasma plume symmetry axes. The fiber-plasma plume distance was adjusted to ensure that the acceptance solid angle of the fiber covers most of the plume emission to avoid any inhomogeneity in the laser- induced plasma (LIP). The output of the optical fiber was connected to an echelle spectrometer (Michelle 7500, Multichannel, Sweden) coupled to an intensified charge coupled device (ICCD) camera (DiCamPro-Computer Optics, Germany), allowing simultaneous spectral analysis in the range of $200-900 \mathrm{~nm}$ with a constant spectral resolution $\lambda / \Delta \lambda=7500$. A gate width of $2500 \mathrm{~ns}$ was chosen for maximizing spectral line intensity while maintaining a good temporal resolution. The plasma emission spectrum was recorded at several delay times (from 0 to $10000 \mathrm{~ns}$ ) at atmospheric pressure. Temporally resolved optical emission spectroscopy is used to investigate the evolution of the spectral line intensity as a function of delay. The gate width and delay time for the spectroscopic data acquisition were controlled by proper computer software.

To optimize the signal-to-noise ratio and spectral reproducibility, the acquisition of the spectra was carried out by averaging 5 single accumulated spectra preceded by two single shots for cleaning the sample surface. To avoid any inhomogeneity in the sample, the average spectra at 5 different positions on the sample are taken in each measurement. The analysis of the emission spectra was accomplished using LIBS ++ software [18].

The obtained spectra were fitted versus the camera sensitivity curve described in the camera manual in order to determine the real intensity of the spectral lines.

The pulse laser energy was measured by a Scientech Joul-meter (model AC5001, USA) and monitored using a fast photodiode and an oscilloscope. The experimental setup is described in detail elsewhere [19].

\section{Results and Discussion}

In order to perform this study, several steps have been carried out. At the beginning the effect of the used detector response throughout the measured spectral range should be considered in order to be able to get the real intensities of the spectral lines. In order to do this, the response curve of the ICCD that was given in its data sheet, was drawn, interpolated to the same number of points as in the LIBS spectra then compensate the camera effect in the spectra by dividing the LIBS spectra on the response curve Fig. (1). 
Table (1): The composition of the cupper alloys used in the study

\begin{tabular}{ccccc}
\hline Sample & Cu $(\%)$ & Zn $(\%)$ & Pb $(\%)$ & Sn $(\%)$ \\
\hline S160 & 85.0 & 2.0 & 6.5 & 6.5 \\
S162 & 93.0 & - & 2.0 & 5.0 \\
S164 & 88.0 & 8.0 & 2.0 & 2.0 \\
\hline
\end{tabular}

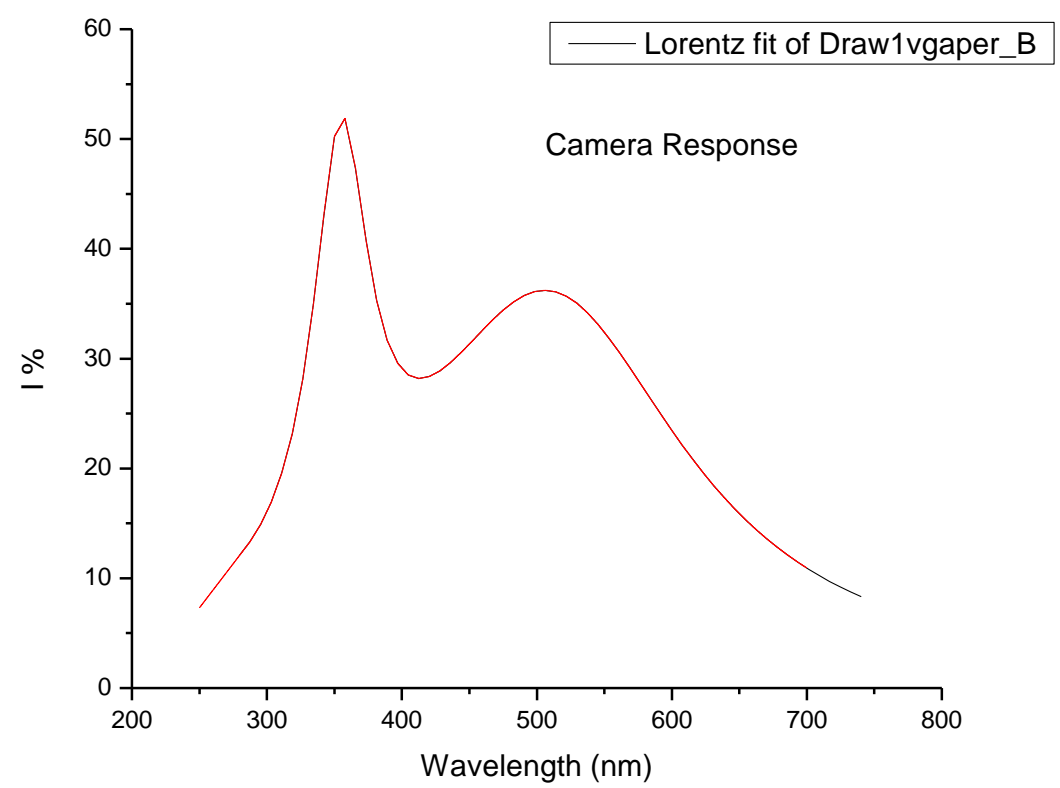

Fig. (1): Response curve of the ICCD

a

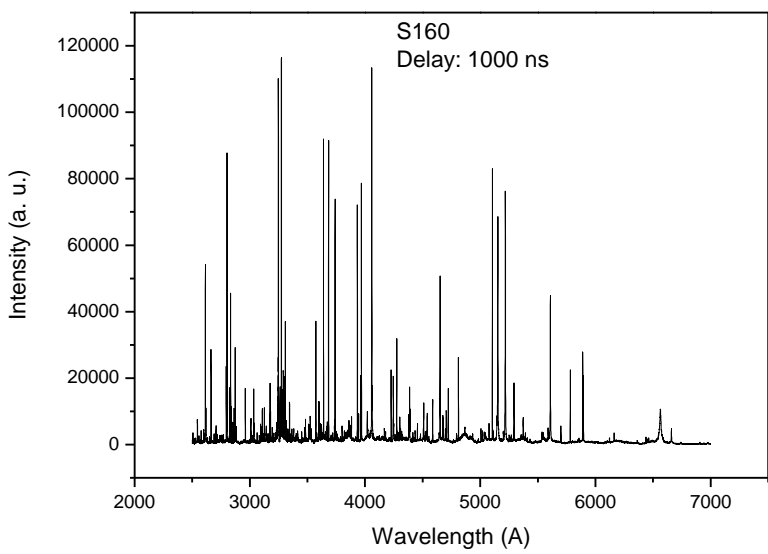

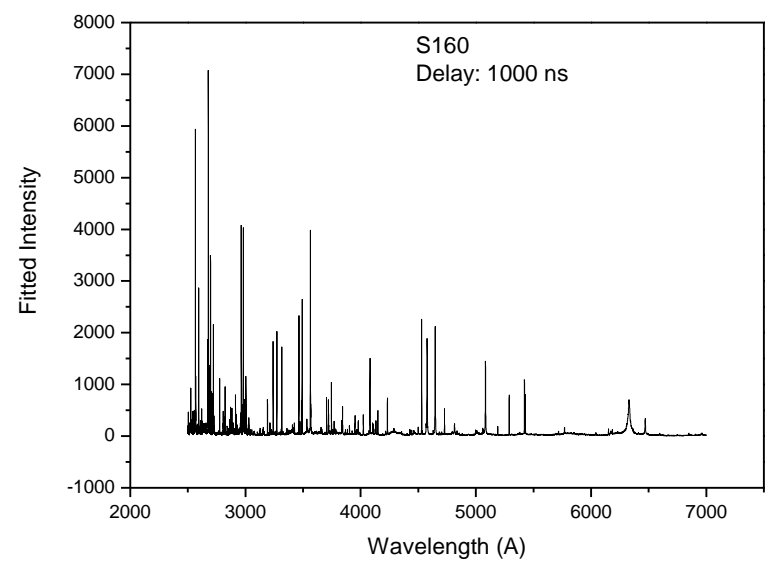

Fig. (2): Sample spectrum a) before and b) after fitting with the camera response curve 
Figure (3) shows the behavior of the intensities of some chosen $\mathrm{Cu}$ spectral lines at different delay times in the three measured $\mathrm{Cu}$ alloys. As it can be seen, as the delay time increases the spectral line intensity decreases for all lines. This was the first landmark showing that there must be a correlation between the spectral lines intensities that may lead us to find a phenomenological formula for the relative intensities for the LIBS spectral lines.

Trying to find the correlation between the spectral lines, the relation between the spectral line intensity for each line and the delay time was plotted. Figure (4) shows the relation between the spectral line intensity and delay time.

On following the Intensity, I of the spectral lines versus the delay time $t$, it was found that it decreases with time exponentially and all the lines follow the same exponential decay equation:
$I=y_{o}+a e^{(-t / b)}$

where $\mathrm{y}_{\mathrm{o}}, \mathrm{a}$ and $\mathrm{b}$ are constants that differs from one line to another. These constants were recorded for each spectral line. Table (3) shows the constants values for the chosen spectral lines.

In order to examine whether all the samples follow a relative intensity or not, sample (S160) was chosen as a reference sample since it has the least $\mathrm{Cu}$ concentration. For each line in the reference sample, the fitting line will be assumed to be the relative intensity for this line, then for the other samples the behavior of each line will be compared to the behavior of its assumed relative intensity. This comparison will be performed by dividing the obtained line intensity by its assumed relative intensity in the reference sample. If the ratios between the spectral lines revealed a constant value for all samples, then they have a relative intensity that can be tabulated.

Table (2): The copper lines used in the present study with their spectroscopic parameters

\begin{tabular}{|c|c|c|c|c|c|}
\hline Wavelength & specie & $\mathbf{E}_{\mathrm{k}}\left(\mathrm{cm}^{-1}\right)$ & $\mathbf{A}_{\mathbf{k i}}\left(\mathbf{s}^{-1}\right)$ & $\mathbf{g}_{\mathrm{k}}$ & $\mathbf{g}_{\mathbf{i}}$ \\
\hline 3035.994 & $\mathrm{Cu}$ & 46172.84 & 0.02428 & 4 & 4 \\
\hline 3063.424 & $\mathrm{Cu}$ & 45879.31 & 0.0155 & 4 & 4 \\
\hline 3194.102 & $\mathrm{Cu}$ & 44544.15 & 0.01549 & 4 & 4 \\
\hline 3524.292 & $\mathrm{Cu}$ & 72093.08 & 0.3286 & 8 & 6 \\
\hline 3598.983 & $\mathrm{Cu}$ & 71290.54 & 1.293 & 10 & 8 \\
\hline 3601.786 & $\mathrm{Cu}$ & 71268.21 & 1.613 & 8 & 8 \\
\hline 4275.251 & $\mathrm{Cu}$ & 62403.32 & 0.3176 & 8 & 6 \\
\hline 4509.659 & $\mathrm{Cu}$ & 64472.3 & 0.2955 & 2 & 4 \\
\hline 4651.306 & $\mathrm{Cu}$ & 62403.32 & 0.4194 & 8 & 10 \\
\hline 4704.878 & $\mathrm{Cu}$ & 62403.32 & 0.06219 & 8 & 8 \\
\hline 5105.655 & $\mathrm{Cu}$ & 30783.69 & 0.01949 & 4 & 6 \\
\hline 5153.033 & $\mathrm{Cu}$ & 49935.2 & 1.034 & 4 & 2 \\
\hline 5218.086 & $\mathrm{Cu}$ & 49942.06 & 1.221 & 6 & 4 \\
\hline 5292.888 & $\mathrm{Cu}$ & 62403.32 & 0.109 & 8 & 8 \\
\hline 5700.313 & $\mathrm{Cu}$ & 30783.69 & 0.002501 & 4 & 4 \\
\hline 5782.234 & $\mathrm{Cu}$ & 30535.3 & 0.019 & 2 & 4 \\
\hline
\end{tabular}



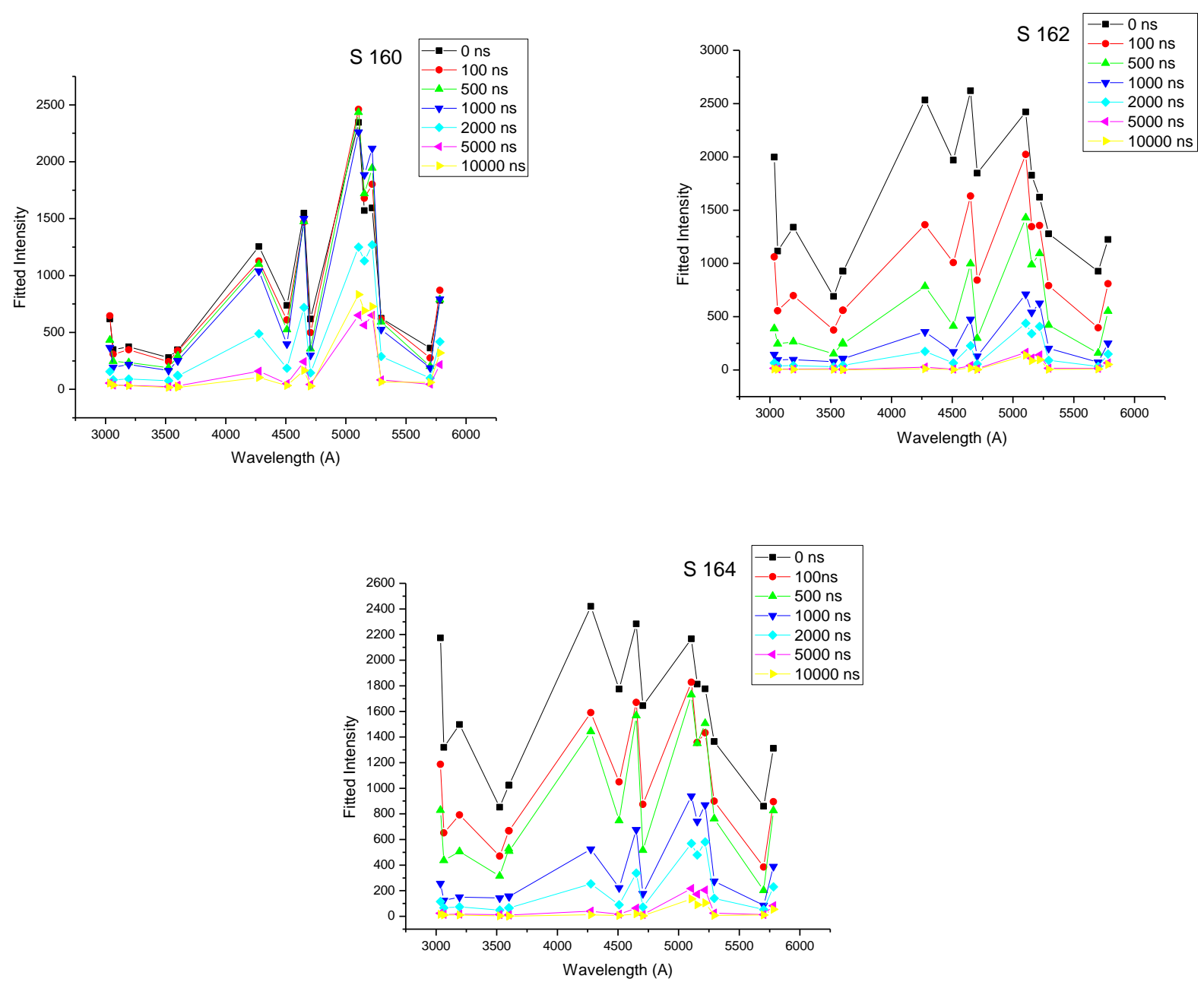

Figure (3): The behavior of the intensities of some chosen $\mathrm{Cu}$ spectral lines at different delays in the three cu alloys
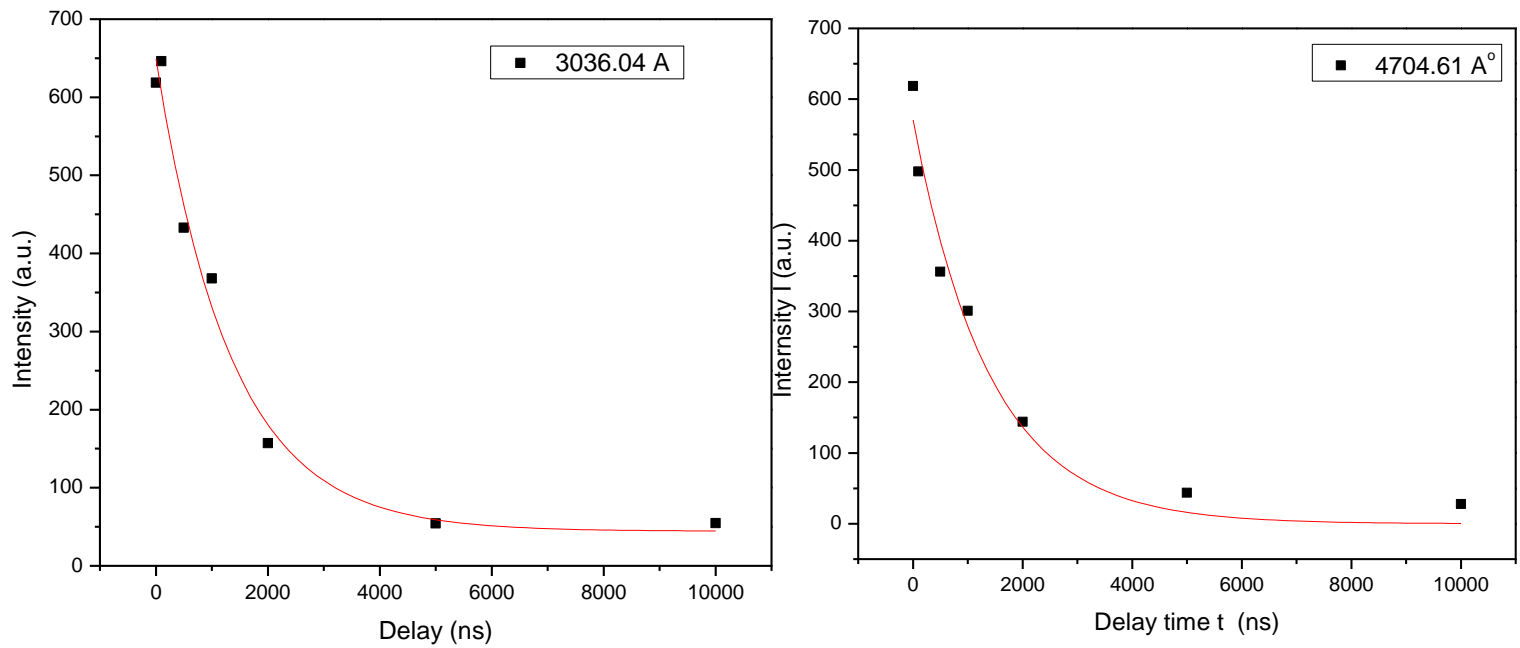

Figure (4): Time evolution of two copper spectral lines

Arab J. Nucl. Sci. \& Applic. Vol. 52, No. 4 (2019) 
Table (3): The exponential line fitting constants of the chosen spectral lines for the sample S160

\begin{tabular}{|c|c|c|c|}
\hline wavelength & $\mathbf{y}_{\mathbf{o}}$ & $\mathbf{a}$ & b \\
\hline 3036.04 & 44.31643 & 604.4508 & 1344.307 \\
\hline 3063.449 & 30.81434 & 312.3791 & 1326.177 \\
\hline 3194.029 & 29.36507 & 340.7927 & 1299.038 \\
\hline 3524.228 & 13.53273 & 255.7916 & 1573.401 \\
\hline 3598.956 & 14.9412 & 342.7083 & 2033.293 \\
\hline 3601.8 & 5.47191 & 351.9949 & 2106.16 \\
\hline 4275.386 & 36.40406 & 1230.755 & 2553.121 \\
\hline 4509.396 & 0 & 701.1881 & 1635.228 \\
\hline 4651.353 & 166.1726 & 1475.539 & 2639.983 \\
\hline 4704.619 & 0 & 570.5869 & 1399.994 \\
\hline 5105.668 & 650.6591 & 1933.663 & 2355.553 \\
\hline 5152.987 & 564.5308 & 1227.424 & 3220.473 \\
\hline 5217.889 & 650.5131 & 1278.876 & 3426.179 \\
\hline 5292.617 & 64.53364 & 599.9254 & 2350.171 \\
\hline 5700.13 & 44.64991 & 284.3798 & 1156.839 \\
\hline 5781.839 & 215.9091 & 661.5591 & 2394.831 \\
\hline
\end{tabular}

Figure (5) shows a relation between the spectral line intensity divided by its relative intensity versus the wave length for different delay times. It can be noticed that there is no constant ratio for all lines, but there are some other observations that can be seen. First of all, the plot shows a linear relationship between the intensity ratio and the wavelength. This indicates that there is some type of correlation between the line intensity ratios. Also, the absolute value of the slope of the fitting line tends to zero with increasing the delay time to $5000 \mathrm{~ns}$, then it deviates again from zero slop for higher delay time. Secondly, Figure 5 also shows that for the delay times lower than $1000 \mathrm{~ns}$, the points are highly scattered from the fitting line of the linear fit and the same occurred again in the delay times higher than 2000 ns. While for the delay time $1000 \mathrm{~ns}$ and $2000 \mathrm{~ns}$ the scattering from the linear fitting line is much smaller. This indicates that, in this delay range, the correlation between the lines is much stronger and has a constant behavior since the slope is of a small value. Thirdly, Figure 5 shows that as the concentration of copper in the sample increases the average intensity of the copper lines decreases.
In order to justify these phenomena taking place in the delay range between $1000 \mathrm{~ns}$ and $2000 \mathrm{~ns}$, the LTE conditions throughout the chosen delay range were examined using McWhirter criterion stating that [20 - 22]:

$\mathrm{N}_{\mathrm{e}}\left(\mathrm{cm}^{-3}\right) \geq 1.6 \times 10^{12} \Delta \mathrm{E} \cdot \mathrm{T}_{\mathrm{e}}^{1 / 2}$

Where: $\mathrm{Ne}$ is the electron density, $\mathrm{Te}$ is the electron temperature and $\Delta \mathrm{E}(\mathrm{eV})$ is the largest energy transition for which the condition holds.

Figure (6) shows the relation between the electron density $\mathrm{N}_{\mathrm{e}}$ and the calculated value of the McWhirter equation. As can be seen, the value of the McWhirter equation is less than that of the $\mathrm{N}_{\mathrm{e}}$ for delay time larger than 3000 ns which indicates that the criterion is fulfilled. As the delay time increases it was found that the value of $\mathrm{N}_{\mathrm{e}}$ decreases to values less than that of the McWhirter equation, which indicates that the LTE conditions are not fulfilled.

It is well known that at the early delay time, the LTE conditions are not fulfilled due to the fact that the expansion time of the plasma is much shorter than the ionization time [23]. So, from the previous data, it could be concluded that the LTE conditions are fulfilled in the delay range between $1000 \mathrm{~ns}$ 
and 2000 ns. This was also confirmed in the work of Galmed and Harith [20]. That is why the range of confidence in this delay time range was the smallest and the data points scattering was low. This indicates that in order to find a correlation between the lines to construct a database for the LIBS spectral lines relative intensities, the LTE conditions should be fulfilled.

On the other hand, it was observed that the range of confidence may be used as an indication for the fulfillment of the LTE conditions.

Figure (5) reveals the inverse proportionality between the concentration of the copper in the sample and the average intensity of the spectral lines. This was also observed in the work of El Hassan et al. [24] and Borisov et al. [25]. This behavior may be related to the change in the physical characteristics of the samples occurring when the $\mathrm{Cu}$ concentration in the standards is varied, and it is one of the most convincing examples of the occurrence of matrix effects in this kind of samples [24,25]. Also, the change in the sample hardness will cause a change in the plasma excitation temperature $\left(\mathrm{T}_{\mathrm{e}}\right)$. $[26,27,28]$

\section{Conclusion}

From the present study, it can be concluded that there is some type of correlation between the LIBS spectral lines, which indicates that there is a possibility for constructing a database for the spectral lines relative intensity. This was proved by plotting the relation between the spectral lines intensity ratio of two alloys of different $\mathrm{Cu}$ concentration versus the wavelength for different delay times, considering that one of them to be an assumed relative intensity.

Also, it has been demonstrated that at the delay time between $1000 \mathrm{~ns}$ and $2000 \mathrm{~ns}$, where the LTE conditions are fulfilled, the scattering from the linear fitting was small and the range of confidence was narrower than in the other delay times which indicate that the relation is much stronger. This also indicates that LTE is a very essential condition for building up the data base for the LIBS spectral lines relative intensity.

It could be observed that the spectral lines intensity ratio between two samples can be considered an evidence for the fulfillment of the LTE conditions through the degree of scattering of the line ratio value.

There is a strong relation between the intensity ratio and the concentration ratio, where it was found that in case of $\mathrm{Cu}$ alloys, the intensity ratio decreases as the $\mathrm{Cu}$ concentration in the alloy increases., which agreed with the observations by El Hassan et al. [24] and Borisov et al [25]. This implies that further future work should be done for more investigations.

\section{Future Work}

More studies should be conducted to get a database for spectral lines relative intensity for LIBS. 

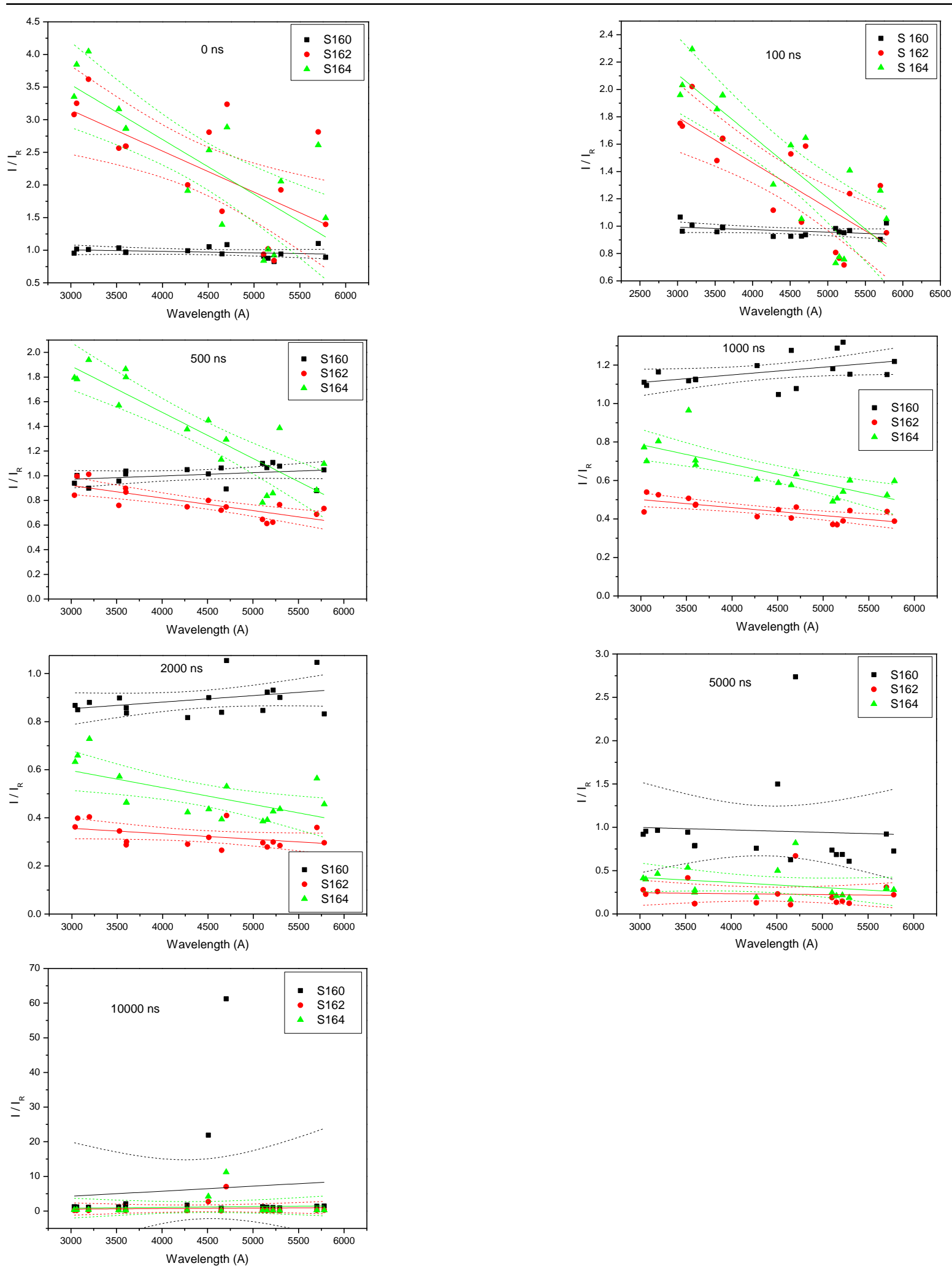

Figure 5 : The relation between the spectral line intensity divided by its relative intensity versus the wave length for different delay times. 


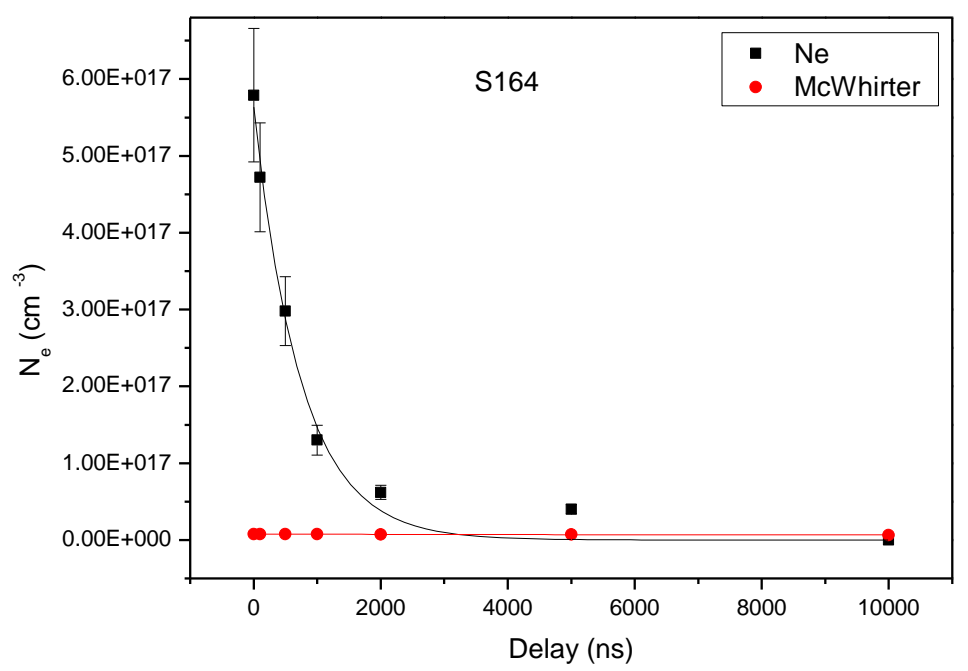

Figure (6): The relation between the electron density $\mathrm{N}_{\mathrm{e}}$ and the calculated value of the McWhirter equation for the sample S164

\section{References}

1. D. A. Cremers and L. J. Radziemski, Handbook of laser-induced breakdown spectroscopy (John Wiley \& Sons Ltd, West Sussex, 2006)

2. V. K. Unnikrishnan, K. Mridul, R. Nayak, K. Alti, V. B. Kartha, C. Santhosh, G. P. Gupta and B. M. Suri, Calibration-free laser induced breakdown spectroscopy for quantitative elemental analysis of materials, PRAMANA journal of Physics 79, 299 ( 2012).

3. R. Gaudiuso, M. Dell'Aglio, O. De Pascale, A. Santagata and A. De Giacomo, Laser-induced plasma analysis of copper alloys based on Local Thermodynamic Equilibrium: An alternative approach to plasma temperature determination and archeometric applications, Spectrochimica Acta Part B 74, 38 (2012).

4. S. Guirado, F. J. Fortes, V. Lazic and J.J. Laserna, Chemical analysis of archeological materials in submarine environments using laser-induced breakdown spectroscopy. On-site trials in the Mediterranean Sea, Spectrochimica Acta Part B 74, 137 (2012).

5. R. S. Harmon, R. E. Russo and R. R. Hark, Applications of laser-induced breakdown spectroscopy for geochemical and environmental analysis: A comprehensive review, Spectrochimica Acta B 87, 11 (2013).

6. David M. Benton, Identification of Asbestos Using Laser-Induced Breakdown Spectroscopy: A Viable Alternative to the

$$
\begin{aligned}
& \text { Conventional Approach?, } \\
& \text { Spectroscopy 2013, } 6 \text { (2013). }
\end{aligned}
$$

7. R. Gaudiuso, M. Dell'Aglio, O. De Pascale, G. S. Senesi and A. De Giacomo, Laser Induced Breakdown Spectroscopy for Elemental Analysis in Environmental, Cultural Heritage and Space Applications: A Review of Methods and Results, Sensors10, 7434 ( 2010).

8. G.S. Senesi, M. Dell'Aglio, R. Gaudiuso, A. De Giacomo, C. Zaccone, O. De Pascale, T. M. Miano, M. Capitelli, Heavy Metal Concentrations in Soils as determined by Laser induced Breakdown Spectroscopy (LIBS), with Special Emphasis on Chromium, Environ. Res.109, 413( 2009).

9. J. B. Sirven, B. Bousquet, L. Canioni and L. Sarger, Laser-induced Breakdown Spectroscopy of Composite Samples: Comparison of Advanced Chemometrics Methods, Anal. Chem. 78, 1462 (2006).

10. J. Amador-Hernández, L. E. García-Ayuso, J. M. Fernandez-Romero and M. D. Luque de Castro, Partial Least Squares Regression for Problem Solving in Precious Metal Analysis by Laser Induced Breakdown Spectrometry, J. Anal. At. Spectrom. 15, 587 (2000).

11. J. El Haddad, D. Bruyère, A. Ismaël, G. Gallou, V. Laperche, K. Michel, L. Canioni, B. Bousquet, Application of a series of artificial neural networks to on-site quantitative analysis of lead into real soil samples by laser induced breakdown 
spectroscopy, Spectrochimica Acta Part B 97, 57 (2014).

12. B. Bousquet, G. Travaille, A. Ismael, L. Canioni, K. Michel-Le Pierres, E. Brasseur, S. Roy, I. Le Hecho, M. Larregieu, S. Tellier, M. Potin-Gautier, T. Boriachon, P. Wazen, A. Diard and S. Belbeze, Development of A Mobile System based on Laser-induced Breakdown Spectroscopy and Dedicated to in situ Analysis of Polluted Soils, Spectrochim. Acta B 63, 1085 (2008).

13. S.N. Panya panya, A.H. Galmed, M. Maaza, B.M. Mothudi, M. A. Harith, J. Kennedy, Laser-Induced Breakdown Spectroscopy (LIBS) on Geological Samples: Compositional Differentiation, MRS Advances 3, 1969 ( 2018).

14. Ping Yang, Ran Zhou, Wen Zhang, Shisong Tang, Zhongqi Hao, Xiangyou Li, Yongfeng Lu, and Xiaoyan Zeng, Laser-induced breakdown spectroscopy assisted chemometric methods for rice geographic origin classification, Applied Optics 57, 8297 (2018).

15. M. M. Tripathi, K. E. Eseller, F. -Y. Yueh and J. P. Singh, Multivariate Calibration of Spectra Obtained by Laser Induced Breakdown Spectroscopy of Plutonium Oxide Surrogate Residues, Spectrochim. Acta B 64, 1212 (2009).

16. A. Ciucci, M. Corsi, V. Palleschi, S. Rastelli, A. Salvetti and E. Tognoni, New Procedure for Quantitative Elemental Analysis by Laser Induced Plasma Spectroscopy, Appl. Spectrosc. 53, 960 (1999).

17. E. Tognoni, G. Cristoforetti, S. Legnaioli and V. Palleschi, Calibration-Free Laser-induced Breakdown Spectroscopy: State of the Art. Spectrochim. Acta B 65, 1 (2010).

18. G.H. Cavalcanti a, D.V. Teixeira a, S. Legnaioli b, G. Lorenzetti b, L. Pardini b, V. Palleschi, One-point calibration for calibrationfree laser-induced breakdown spectroscopy quantitative analysis, Spectrochimica Acta Part B 87, 51 (2013).

19. M. Sabsabi, V. Detalle, M.A. Harith,W. Tawfik and H. Imam, Comparative study of two new commercial echelle spectrometers equipped with intensified CCD for analysis of laser-induced breakdown spectroscopy, Appl. Opt. 42, 6094 (2003).

20. A.H. Galmed and M.A. Harith, Temporal follow up of the LTE conditions in aluminum laser induced plasma at different laser energies, Appl. Phys. B 91, 651 (2008).

21. R. W. P. McWhirter, in Plasma Diagnostic Techniques, R. H. Huddlestone and S. L. Leonard, Eds. (Academic Press, New York, 1965), Chap. 5, pp. 201-264.
22. G. Cristoforetti, A. De Giacomo, M. Dell'Aglio, S. Legnaioli, E. Tognoni, V. Palleschi, and N. Omenetto, Spectrochim Acta, Part B 65, 86 (2010).

23. O. Barthe'lemy, J. Margot, S. Laville, F. Vidal, M. Chaker, B. Le Drogoff, T. W. Johnston, and M. Sabsabi, Investigation of the State of Local Thermodynamic Equilibrium of a Laser Produced Aluminum Plasma, Appl. Spectrosc. 59, 529 (2005).

24. A. Elhassan, G. Cristoforetti, S. Legnaioli, V. Palleschi, A. Salvetti, E. Tognoni, G. Ingo, M. A. Harith, LIBS Calibration Curves and Determination of Limits of Detection (LOD) in Single and Double Pulse Configuration for Quantitative LIBS Analysis of Bronzes, CSSIM / ICOM-LIC Meeting 2007, Cairo, p72.

25. O. V. Borisov, , X. L. Mao, , A. Fernandez, , M. Caetano and R. E. Russo, Inductively Coupled Plasma Mass Spectrometric Study of Non-Linear Calibration Behavior During Laser Ablation of Binary $\mathrm{Cu}-\mathrm{Zn}$ Alloys, Spectrochimica Acta B 54, 1351 (1999).

26. K. Tsuyuki, S. Miura, N. Idris, K. Hendrik, T. Jie, K. Kagawa, Measurement of concrete strength using the emission intensity ratio between $\mathrm{Ca}$ (II) $396.8 \mathrm{~nm}$ and $\mathrm{Ca}(\mathrm{I}) 422.6 \mathrm{~nm}$ in a Nd:YAG laser induced plasma. Appl. Spectrosc. 60, 61 (2006).

27. Z.A. Abdel-Salam, A.H. Galmed, E. Tognoni, M.A. Harith, Estimation of calcified tissues hardness via calcium and magnesium ionic to atomic line intensity ratio in laser induced breakdown spectra. Spectrochim. Acta B 62, 1343 (2007).

28. A. H. Galmed, C. Steenkamp, I. Ahmed, A. du Plussis, H. von Bergmann, M. A. Harith, M. Maaza, Using laser-induced breakdown spectroscopy to monitor the surface hardness of titanium samples bombarded by carbon ions, Applied Physics B 124 (2018). 